\section{(A) Check for updates}

Cite this: Polym. Chem., 2021, 12 , 5377

Received 13th July 2021,

Accepted 5th September 2021

DOI: $10.1039 /$ d1py00952d

rsc.li/polymers

\title{
Fully amorphous atactic and isotactic block copolymers and their self-assembly into nano- and microscopic vesicles $\dagger$
}

\author{
Riccardo Wehr, ${ }^{a}$ Elena C. dos Santos, ${ }^{a}$ Moritz S. Muthwill, (D) ${ }^{a}$ Vittoria Chimisso, ${ }^{a}$ \\ Jens Gaitzsch (iD *a,b and Wolfgang Meier (iD *a
}

\begin{abstract}
The introduction of chirality into aqueous self-assemblies by employing isotactic block copolymers (BCPs) is an emerging field of interest as it promises special membrane properties of polymersomes not accessible by atactic BCPs. However, isotactic BCPs typically exhibit crystalline behaviour, inducing high membrane stiffness and limiting their applicability in systems involving membrane proteins or sensitive cargo. In this study, an isotactic yet fully amorphous BCP is introduced which overcomes these limitations. Three BCPs composed of poly(butylene oxide)-block-poly(glycidol) (PBO-b-PG), differing solely in their tacticities ( $R / S, R$ and $S$ ), were synthesised and characterised regarding their structural, optical and thermal properties. Their self-assembly into homogenous phases of nanoscopic polymersomes (referred to as small unilamellar vesicles, SUVS) was analysed, revealing stability differences between SUVS composed of the different BCPs. Additionally, microscopic giant unilamellar vesicles (GUVs) were prepared by double emulsion microfluidics. Only the atactic BCP formed GUVs which were stable over several hours, whereas GUVs composed of isotactic BCPs ruptured within several minutes after formation. The ability of atactic PBO- $b-P G$ to form microreactors was elucidated by reconstituting the membrane protein OmpF in the GUV membrane by microfluidics and performing an enzyme reaction inside its lumen. The system presented here serves as platform to design versatile vesicles with flexible membranes composed of atactic or isotactic BCPs. Hence, they allow for the introduction of chirality into nano- or microreactors which is a yet unstudied field and could enable special biotechonological applications.
\end{abstract}

\section{Introduction}

Aqueous self-assembly of amphiphilic block copolymers (BCPs) into nano- and microscopic vesicles is an established strategy to produce compartments used in drug delivery, as nanoreactors or cell mimics. ${ }^{1-5}$ When employing BCPs bearing side groups on their backbones, such as poly( $\mathrm{N}$-(2-hydroxypropyl)methacrylamide) (PHPMA) or poly(propylene oxide) (PPO), these are usually in their atactic configuration. Only recently, the use of isotactic hydrophobic blocks has attracted increasing interest. ${ }^{6-12}$ In particular, isotactic poly(lactic acid) (PLA) has been used to elucidate the effect of stereoregularity in the main chain of BCPs on the formation and morphology of

\footnotetext{
${ }^{a}$ University of Basel, Department of Chemistry, Mattenstrasse 24a, BPR 10964058 Basel, Switzerland. E-mail: gaitzsch@ipfdd.de, wolfgang.meier@unibas.ch

${ }^{b}$ Leibniz-Institut für Polymerforschung Dresden e.V., Hohe Strasse 6, 01069 Dresden, Germany

$\dagger$ Electronic supplementary information (ESI) available: Details about materials and syntheses, polymer and self-assembly characterisation as well as CLSM images of the enzyme reaction within PDMS- $b$-PMOXA GUVs. In addition, videos of time lapses of $(R / S)$-BCP GUVs with and without OmpF and PDMS- $b$-PMOXA GUVs with and without OmpF. See DOI: 10.1039/d1py00952d
}

aqueous self-assemblies. For example, poly(L-lactic acid)-blockpoly(acrylic acid) (PLLA- $b$-PAA) BCPs formed cylindrical micelles, whereas spherical micelles were obtained when atactic PLA was employed. ${ }^{7,8}$ Differences between atactic and isotactic PLA- $b$-PEG self-assemblies were not observed regarding the morphology of spherical micelles, cylindrical micelles and vesicles; only the packing of chains within the vesicle membrane was affected, as isotactic BCPs showed a denser and more elongated conformation within the membrane. ${ }^{12}$

In general, all of the aforementioned studies were based on crystallisation-driven self-assembly (CDSA). Thus, the isotacticity of the PLLA block served solely as means to introduce crystallinity within the membrane. Hence, all differences between self-assemblies composed of isotactic PLLA and their counterparts composed of atactic, amorphous PLA can be explained by the presence of crystallinity. However, this prevents a higher complexity and applicability as crystalline membranes generally hamper applications requiring highly flexible and fluid membranes, such as the functional reconstitution of membrane proteins. ${ }^{13,14}$ Additionally, the need to thoroughly dilute crystalline BCPs or to heat them above their melting or glass transition temperatures $\left(T_{\mathrm{g}}\right)$ to form well-controlled self-assem- 
blies prevents a combination with cargo sensitive to organic solvents or elevated temperatures. ${ }^{15-17}$ Advanced applications such as platforms to analyse enzymatic cascade reactions or cell mimics with controlled passage of substrates are thus considerably more difficult with (semi-) crystalline BCPs. ${ }^{18-23}$

Polymeric self-assemblies composed of isotactic, yet noncrystalline, BCPs to create fully flexible and amorphous chiral membranes would overcome these limitations but have not been available so far. In this work, a straightforward approach to obtain small and giant unilamellar vesicles (SUVs, GUVs) composed of fully amorphous isotactic BCPs is presented. Poly (butylene oxide)-block-poly(glycidol) (PBO- $b$-PG) was chosen as vesicle-forming BCP, since its atactic version fulfills all requirements to prepare biomedically applicable nanoparticles: ${ }^{24,25}$ both blocks are biocompatible, fully amorphous ${ }^{26-28}$ and their side groups allow for the introduction of isotacticity. Here, a set of three amphiphilic PBO- $b$-PG BCPs with either atactic $(R /$ $S)$, isotactic $(R)$ or isotactic $(S)$ configuration, was synthesised. All BCPs were characterised thoroughly in bulk and in solution, especially with respect to their chiral properties and in comparison with the respective homopolymers of both blocks. SUVs were characterised in aqueous medium and differences between SUVs composed of atactic and isotactic BCPs regarding formation, morphology, stability and membrane thickness were investigated. Additionally, GUVs of all BCPs were prepared using double emulsion microfluidics. As a proof of concept, the bacterial Outer membrane protein $\mathrm{F}(\mathrm{OmpF})$ was inserted into the membrane of atactic GUVs and its successful functional reconstitution was proven by an enzyme reaction. This work represents the first study separating the effects of chirality and crystallinity on aqueous self-assemblies. It allows to assess how BCP tacticity affects the formation, morphology, stability and membrane thickness of SUVs and GUVs without affecting the membrane flexibility. In turn, this will be crucial for a future combination with membrane proteins reconstituted in chiral polymer membranes.

\section{Experimental section}

The used materials and the synthesis details are reported in the ESI. $\dagger$

\subsection{Methods}

Nuclear magnetic resonance (NMR) spectroscopy. ${ }^{1} \mathrm{H}$ and ${ }^{13} \mathrm{C}$ NMR spectra were recorded on an NMR spectrometer (500 MHz, Avance III, Bruker, USA) in methanol-d $\mathrm{d}_{4}$ (MeOD) at $25{ }^{\circ} \mathrm{C}$. Each sample was measured with a default number of 16 scans for the ${ }^{1} \mathrm{H}$ NMR spectra and 1024 scans for the ${ }^{13} \mathrm{C}$ NMR spectra. Calibration was performed in reference to the $\mathrm{H}_{2} \mathrm{O}$ signal in MeOD (4.85 ppm). The spectra were processed and analysed using Mestrenova 11.0 software (Mestrelab, Spain).

Size exclusion chromatography (SEC). SEC meausurements were run on an SEC device equipped with three GRAM columns (1000 ̊, $10 \mu \mathrm{m}, 8 \times 300 \mathrm{~mm}$, PSS, Germany), a PSS precolumn (GRAM, $5 \mu \mathrm{m}, 8 \times 50 \mathrm{~mm}$ ) and a refractive index
(RI) detector (Agilent 1260 Infinity II, USA). Poly(ethylene glycol) molecular weight standards of narrow dispersities were used for calibration. The measurements were preformed in HPLC grade DMF (Scharlau, Germany) at $60^{\circ} \mathrm{C}$ with a flow rate

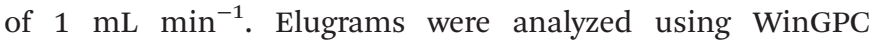
UniChrom software (version 8.33, PSS).

Polarimetry. Optical rotations were measured on an MCP 100 (Anton Paar, Austria), equipped with a $589 \mathrm{~nm}$ laser. The stainless steel measurement cylinder had a capacity of $0.05 \mathrm{~mL}$ with a path length of $2.5 \mathrm{~mm}$ and a diameter of $5 \mathrm{~mm} .3$ repetitive measurements were performed at $25{ }^{\circ} \mathrm{C}$, using methanol as solvent and reference, and a monomer or polymer concentration of $50 \mathrm{mg} \mathrm{mL}^{-1}(5 \% \mathrm{w} / \mathrm{v})$. The specific rotation is stated and was calculated from the measured optical rotation $\alpha$ as follows: $[\alpha]_{\mathrm{D}}^{25}=\alpha /(l \cdot c)$ with $[\alpha]_{\mathrm{D}}^{25}$ specific rotation, $l$ path length and $c$ concentration.

Circular dichroism (CD) spectroscopy. CD spectra were recorded on a J-1500 CD spectrometer (Jasco, Japan) equipped with a nitrogen purging set-up and an argon lamp. Measurements were carried out at $20^{\circ} \mathrm{C}$ in a $1 \mathrm{~mm}$ optical path length quartz cuvette (Hellma Analytics, Germany). The spectra were recorded at a sample concentration of $60 \mathrm{mg}$ $\mathrm{mL}^{-1}$ from $600 \mathrm{~nm}$ to $165 \mathrm{~nm}$ at a scan rate of $50 \mathrm{~nm} \mathrm{~min}{ }^{-1}$. $n$-Hexane was used as solvent for the BCPs and PBO homopolymers and water was used for the PG homopolymers.

Thermogravimetric analysis (TGA). TGA measurements were performed on a TGA 5500 (TA Instruments, USA) in aluminium crucibles. 2-5 $\mathrm{mg}$ of the polymers were heated with a heating rate of $10{ }^{\circ} \mathrm{C} \mathrm{min}^{-1}$ from room temperature to $600{ }^{\circ} \mathrm{C}$ under nitrogen atmosphere. The thermograms were analysed using the TRIOS software (TA Instruments, version 5.1.1).

Differential scanning calorimetry (DSC). DSC curves were recorded on a DSC 214 Polyma (Netzsch, Germany) under inert atmosphere $\left(\mathrm{N}_{2}\right)$. All runs were performed at temperatures ranging from $-150{ }^{\circ} \mathrm{C}$ to $120{ }^{\circ} \mathrm{C}$ with a heating rate of $10{ }^{\circ} \mathrm{C}$ $\mathrm{min}^{-1}$. Each sample (10-15 mg) was weighed into aluminium crucibles. Thermograms were analysed using Proteus 7.1 software (Netzsch). Thermal transitions were determined from the second heating curves and the $T_{\mathrm{g}}$ was determined from the inflection point of each curve.

Transmission electron microscopy (TEM). TEM images were recorded on a CM100 TEM (Philips, The Netherlands) at an acceleration voltage of $80 \mathrm{kV} .5 \mu \mathrm{L}$ of a $0.5 \mathrm{mg} \mathrm{mL}^{-1}$ dispersion were deposited on glow-discharged Formvar-coated 200 mesh copper grids for $60 \mathrm{~s}$, blotted off and stained with a $2 \%$ uranyl acetate solution.

Cryogenic TEM (Cryo-TEM). Cryo-TEM images were recorded on a Talos electron microscope (Thermo Fisher, USA) equipped with a CETA camera using a Gatan 626 Cryo-holder. $4 \mu \mathrm{L}$ of a $5 \mathrm{mg} \mathrm{mL} \mathrm{mL}^{-1}$ self-assembly dispersion was adsorbed onto a holey carbon-coated grid (Lacey, Tedpella, USA). The sample was vitrified into liquid ethane using a Leica GP plunger (Leica, Austria). An acceleration voltage of $200 \mathrm{kV}$ and a nominal magnification of $57000 \times$ was used. Membrane thicknesses of 50-100 SUVs per BCP were measured using ImageJ software (NIH, USA). 
Dynamic and static light scattering (DLS/SLS). Multi-angle light scattering data were recorded on an LS spectrometer (LS Instruments, Switzerland) equipped with a $633 \mathrm{~nm} \mathrm{He}-\mathrm{Ne}$ laser (21 $\mathrm{mW})$, ALV correlator and thermostat (Jasco, Japan). Samples were measured at $0.05 \mathrm{mg} \mathrm{mL}^{-1}$ at scattering angles between $30^{\circ}$ and $135^{\circ}$ at $25^{\circ} \mathrm{C}$. DLS was used to calculate the hydrodynamic radius $\left(R_{\mathrm{h}}\right)$ as the mean value of three independent $60 \mathrm{~s}$ measurements obtained by second-order cumulant analysis. The polydispersity index (PDI) was obtained as a mean from all angles. SLS data were used to calculate the radius of gyration $\left(R_{\mathrm{g}}\right)$ either by Guinier or by Mie fitting, depending on size and dispersity of the sample. When using the Guinier model, the natural logarithm of the intensity $(\ln I)$ was plotted against the scattering vector $\left(q^{2}\right)$ in the Guinier regime $\left(30-55^{\circ}\right)$ and fitted with a linear fit. The $R_{\mathrm{g}}$ was calculated from its slope following the equation slope $=-R_{\mathrm{g}}{ }^{2} / 3$. When using the Mie model, the mean intensity of three repetitive measurements was plotted against the corresponding angle and fitted with a Mie scattering model (MiePlot, UK) for $\eta=1.35$ and $5 \%$ polydispersity. The radius $R$ was obtained from the best fit and $R_{\mathrm{g}}$ was calculated from the hard sphere model equation $R_{\mathrm{g}}{ }^{2}=(3 / 5) R^{2}$.

Nanoparticle tracking analysis (NTA). NTA measurements were performed on a NanoSight NS300 (Malvern Panalytical, UK) equipped with a $488 \mathrm{~nm}$ laser and analysed using the NTA software (version 3.4). The self-assemblies $\left(5 \mathrm{mg} \mathrm{mL}^{-1}\right)$ were diluted by a factor of 10000 and injected at a flow rate of

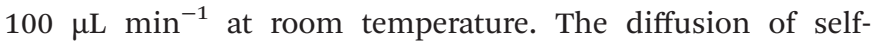
assemblies was recorded over $60 \mathrm{~s}$ in five repetitive measurements. The hydrodynamic radii and their distributions were calculated from the diffusion using the Einstein-Stokes equation and averaged over all measurements.

Confocal laser scanning microscopy (CLSM). CLSM images were recorded using a Zeiss 880 confocal laser scanning microscope (Zeiss, Germany), equipped with a Plan-Apochromat 20×/ 0.8 M27 objective and run by Zen Black software (Zeiss). BODIPY 630/650 dye was excited with a $633 \mathrm{~nm}$ He-Ne laser and resorufin was excited with a $561 \mathrm{~nm}$ diode pumped (DPS) laser. Images were recorded with an image size of $1024 \times 1024$ pixels, a bit depth of 16 bit and 1 Airy unit. Laser power and detector gain were kept constant for all measurements in order to enable fluorescence intensity comparisons between different images. Editing of the images and measuring the diameters of GUVs was done with the ImageJ software (NIH, USA), whereas fluorescence intensities were measured in Zen Blue software (Zeiss).

\subsection{Syntheses}

A Biotage Initiator microwave reactor (Biotage, Sweden) equipped with Robot Eight and an infrared sensor was used to synthesise all polymers. Each polymerisation was carried out at low absorption level after $10 \mathrm{~s}$ prestirring.

The atactic and isotactic poly(butylene oxide)-block-poly(glycidol) (PBO- $b$-PG) BCPs were synthesised in two sequential, microwave-based anionic ring-opening polymerisations following a previously published protocol. ${ }^{25}$ The polymer configuration was predetermined using either racemic or enantiopure butylene oxide (BO) and 1-ethoxyethyl glycidyl ether (EEGE) monomers. The detailed synthetic protocols for all polymers can be found in the ESI. $\uparrow$ Briefly, potassium tert-butoxide solution was transferred into a microwave vial inside a glovebox. After adding the respective BO monomer, the solution was heated to $70{ }^{\circ} \mathrm{C}$. After $34 \mathrm{~min}$, the reaction was quenched by adding methanol. The crude PBO homopolymer was purified by extraction. The chain extension was performed by dissolving the $\mathrm{PBO}$ homopolymer in a microwave vial inside a glovebox. After deprotonation of the terminal hydroxy groups with potassium naphthalenide solution and addition of the respective EEGE monomer, the polymerisation was performed at $70{ }^{\circ} \mathrm{C}$ for $2.5 \mathrm{~h}$. After quenching with methanol, the acetal-protecting groups of the PEEGE blocks were cleaved with $0.1 \mathrm{M}$ HCl. The crude BCP was then purified by dialysis and lyophilisation.

\subsection{Formation of SUVs and GUVs}

Self-assembly into nanoscopic SUVs was done by solvent exchange at a BCP concentration of $5 \mathrm{mg} \mathrm{mL} \mathrm{m}^{-1}$ at room temperature. $10 \mathrm{mg}$ of the copolymer were dissolved in $0.4 \mathrm{~mL}$ THF in a $5 \mathrm{~mL}$ flask. $1.6 \mathrm{~mL}$ phosphate-buffered saline (PBS) were added under stirring at $300 \mathrm{rpm}$ at an addition rate of $10 \mu \mathrm{L}$ $\min ^{-1}$. After stirring overnight, the organic solvent was removed by dialysis against PBS using a regenerated cellulose membrane (MWCO 1 kDa, RC6, Spectra Por, USA). PBS was exchanged four times within two days. All SUV dispersions were extruded using a Mini Extruder (Avanti, USA) equipped with a $100 \mathrm{~nm}$ polycarbonate membrane in 15 passages and characterised before and after extrusion.

Microscopic GUVs were assembled from double emulsion templates created by microfluidics. Water-oil-water double emulsions were generated in a six-way junction microfluidic chip, ${ }^{22}$ with defined flow rates using a three-module precision syringe pump (low pressure NEMESYS, Cetoni, Germany). PBS containing $20 \mathrm{wt} / \mathrm{v} \%$ poly(ethylene glycol) (PEG, $3000 \mathrm{Da})$ and $300 \mathrm{mM}$ sucrose was used as inner aqueous phase (IA). This phase was enveloped by the polymer organic phase (PO), consisting of $4 \mathrm{mg} \mathrm{mL}^{-1}$ of the specific BCP dissolved in 3 : 2 hexane: cholorform (v:v), and subsequently broken up into double emulsions by the outer aqueous phase (OA), composed of $5 \mathrm{wt} / \mathrm{v} \%$ poly(vinyl alcohol) (PVA, 18-23 kDa), $300 \mathrm{mM} \mathrm{NaCl}$ and $0.5 \%(\mathrm{w} / \mathrm{v})$ Pluronic ${ }^{\circledR}$ F-68. The flow rates for double emulsion formation were generally set to $2 \mu \mathrm{L} \min ^{-1}$ (IA), $1 \mu \mathrm{L}$

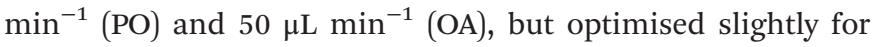
every batch. Live imaging of this process was followed using a high-speed digital microscope (Meros, Dolomite). In order to evaporate the organic solvents from the double emulsions and thus form a vesicular bilayer membrane according to an established protocol, ${ }^{22} 198 \mu \mathrm{L}$ of a double emulsion sample and $2 \mu \mathrm{L}$ of a $100 \mu \mathrm{M}$ aqueous BODIPY 630/650 solution were transferred into a Nunc Lab-Tek eight-well chamber plate (Thermo Fisher Scientific, USA) and incubated at room temperature for $20 \mathrm{~min}$. Within this time, GUVs were created from double emulsions, which settled to the bottom of the well for further CLSM imaging. 


\subsection{OmpF reconstitution and enzyme reaction}

For the enzymatic microreactors, $\beta$-galactosidase $(\beta$-Gal) at a concentration of $0.25 \mathrm{mg} \mathrm{mL}^{-1}$ was added to the IA and $20 \mu \mathrm{L}$ of the Outer membrane protein $\mathrm{F}(\mathrm{OmpF})$ solution at $69.5 \pm$ $0.3 \mu \mathrm{M}$ monomer concentration was added to $1 \mathrm{~mL}$ of the OA. For the negative control, no OmpF was added to the OA. The procedures for expression and purification of OmpF can be found in the ESI. $\dagger 180 \mu \mathrm{L}$ of the GUV dispersion were transferred into a Nunc Lab-Tek eight-well chamber plate (Thermo Fisher Scientific, USA) and $10 \mu \mathrm{L}$ of a proteinase $\mathrm{K}$ solution $\left(1 \mathrm{mg} \mathrm{mL}{ }^{-1}\right)$ was added. After $20 \mathrm{~min}, 10 \mu \mathrm{L}$ of a resorufin $\beta$-Dgalactopyranoside solution (RGP, $53 \mathrm{mM}$ ) was added and a CLSM timelapse over $2 \mathrm{~h}$ was started, recording one image per minute.

\section{Results and discussion}

\subsection{Synthesis of atactic and isotactic homopolymers and} BCPs

The microwave-assisted anionic ring-opening polymerisation of atactic and isotactic PBO- $b$-PG BCPs followed a previously established procedure. ${ }^{25}$ This involved three steps, as displayed in Fig. 1a: (1) synthesis of a hydrophobic PBO homopolymer, which served as a precursor for the following chain extension; (2) chain extension with a protected glycidol derivative, EEGE, to obtain PBO- $b$-PEEGE; (3) acidic cleavage of the protecting groups of the PEEGE block, resulting in the final amphiphilic PBO- $b$-PG. Three similar BCPs were synthesised in this manner, differing only in their configuration (Fig. 1b): one fully atactic PBO- $b-\mathrm{PG}$, referred to as $(R / S)$-BCP, and two all-isotactic PBO- $b$-PGs; one of them in $(R)$ configuration on all repeating units of both blocks, referred to as $(R)$-BCP, and one in $(S)$ configuration, referred to as $(S)$-BCP. The stereocontrol was achieved solely by using racemic or enantiopure monomers of both blocks. Additionally, PG homopolymers of all three configurations were synthesised following a similar protocol (ESI $\dagger$ ). Thus, a series of nine atactic and isotactic polymers - three PBO homopolymers, three PG homopolymers and three PBO- $b$-PG BCPs in $(R / S),(R)$ and $(S)$ configuration were obtained (Table 1 ).

It should be noted that all compounds of one configuration are consistently referred to as $(R)$ or $(S)$. For example, for protected compounds annotated $(R)$-EEGE and $(R)$-PEEGE, the official configuration according to IUPAC would be $(S)$-EEGE and $(S)$-PEEGE, respectively, because of the changing priorities of the side groups compared to their unprotected $(R)$-glycidol precursors and $(R)-\mathrm{PG}$ successors. For the sake of clarity and simplicity, the selected annotation will be used throughout, referring to the related compounds of $(R)$-glycidol and $(R)-\mathrm{PG}$

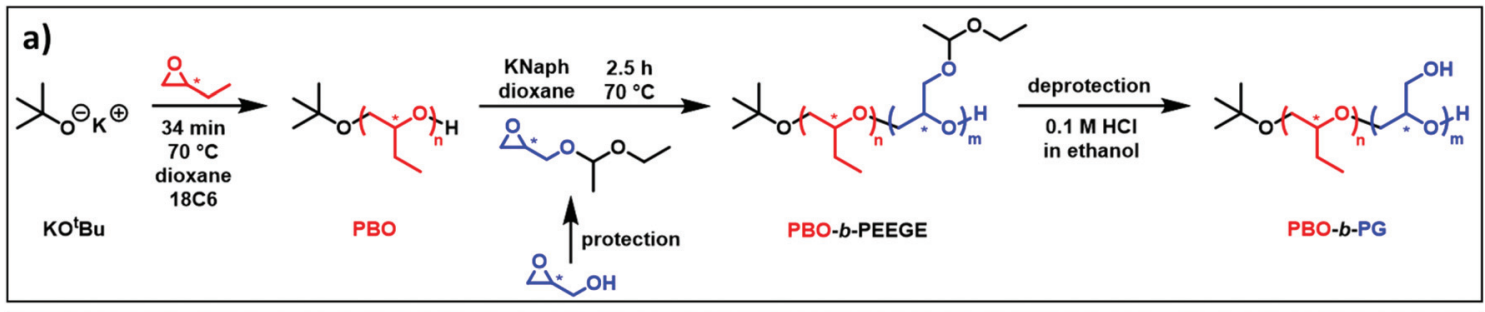

b)

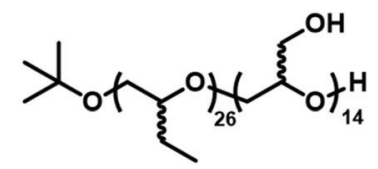

$(R / S)-\mathrm{BCP}$

$(R / S)-\mathrm{PBO}_{26}-b-(R / S)-\mathrm{PG}_{14}$

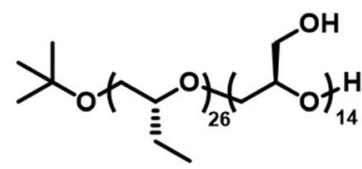

$(R)-\mathrm{BCP}$

$(R)-\mathrm{PBO}_{26}-b-(R)-\mathrm{PG}_{14}$

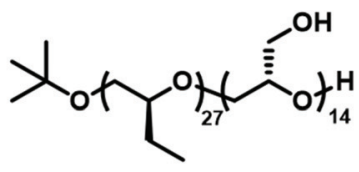

(S)-BCP

$(S)-\mathrm{PBO}_{27}-b-(S)-\mathrm{PG}_{14}$ c)

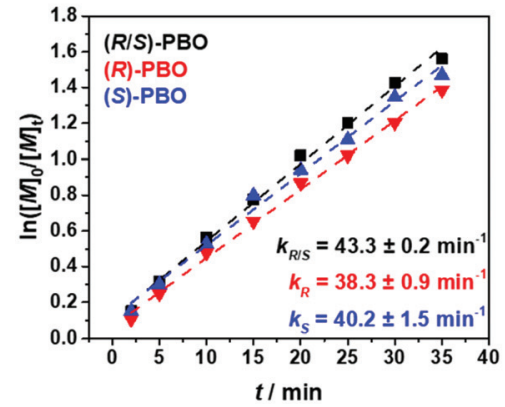

d)

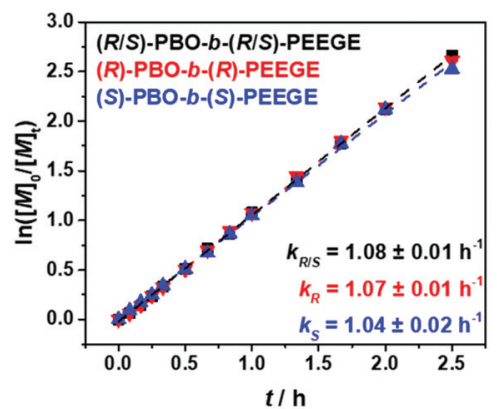

Fig. 1 BCP synthesis. (a) Reaction equation for the syntheses of atactic and isotactic PBO- $b$-PG. The asterisk indicates the stereocentres in every repeating unit of both blocks. (b) Structures and composition of the atactic and isotactic BCPs: $(R / S)$ - $\mathrm{BCP}$ (left), $(R)-\mathrm{BCP}(\mathrm{middle})$ and $(S)$ - $\mathrm{BCP}$ (right). Kinetics of (c) the PBO syntheses and (d) the PBO- $b$-PEEGE syntheses. The reaction constants $k$ are displayed for all three configurations. 
Table 1 Polymer characterisation consisting of composition, ${ }^{a}$ molecular weight $M_{n}{ }^{a}$ hydrophilic block ratio $f^{a}$ dispersity $\Xi^{b}$ specific rotation $[\alpha]{ }_{25}{ }^{c}$, content of meso diads $m^{d}$ and glass transition temperatures $T_{\mathrm{g}}{ }^{e}$

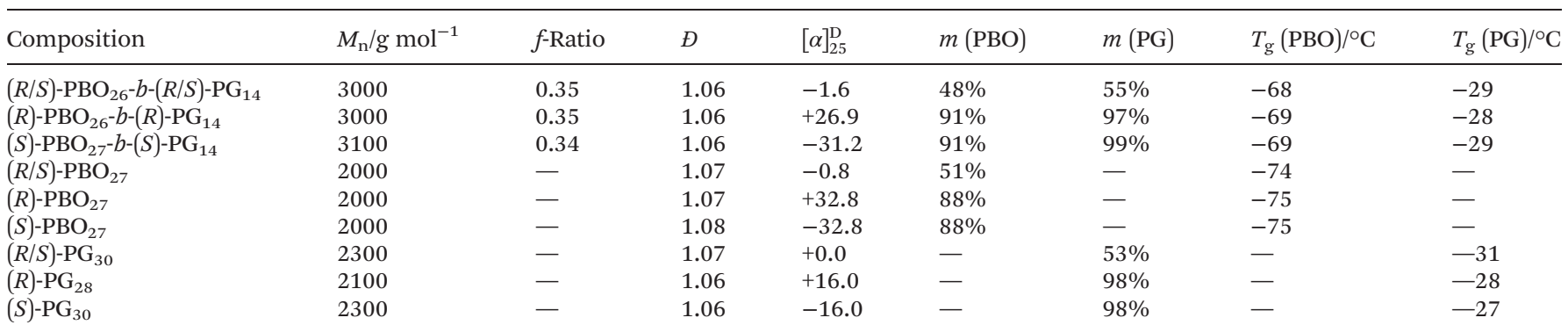

${ }^{a}$ Values determined by ${ }^{1} \mathrm{H}$ NMR spectroscopy. ${ }^{b}$ Values determined by SEC. ${ }^{c}$ Values determined by polarimetry. ${ }^{d}$ Values determined by ${ }^{13} \mathrm{C}$ NMR spectroscopy. ${ }^{e}$ Values determined by DSC.

as $(R)$-EEGE and $(R)$-PEEGE and vice versa for the $(S)$ compounds.

In order to elucidate whether the configuration of the monomers affected the polymerisation process, kinetic measurements were performed (Fig. $1 \mathrm{c}$ and $\mathrm{S} 3-\mathrm{S} 11 \dagger$ ). The kinetics of the PBO syntheses starting from the enantiopure monomers $(R)$ - and $(S)$-BO and of their chain extensions with $(R)$ - and $(S)$-EEGE were analysed and compared to the data obtained for the racemic mixtures. For all polymerisations, ln $\left([M]_{0} /[M]_{t}\right)$ showed a linear increase with the reaction time $t$, representing a living polymerisation and ideal first-order kinetics (Fig. 1c and d). The reaction constant (the slope of the linear fit) was calculated for all PBO and PBO- $b$-PEEGE polymerisations. For the PBO syntheses, values of about $40 \mathrm{~min}^{-1}$ were determined (Fig. $1 \mathrm{c}$ and $\mathrm{S} 3-\mathrm{S} 5 \dagger$ ) and for PBO- $b$-PEEGE values of about $1.05 \mathrm{~h}^{-1}$ (Fig. 1d and S6-S8 $\dagger$ ). Within experimental error, these kinetic constants were independent of the respective configuration. This is evident from the fact that the $(R / S)$-monomer is a racemic mixture of both enantiomers. Hence, it confirmed that atactic PBO and PBO- $b$-PEEGE and thus the final PBO- $b$-PG consisted of statistically distributed $(R)$ and $(S)$ repeating units, as no enantiomer was consumed quicker or slower than the other. The linear growth of the molecular weights with increasing conversion, as well as the low dispersities during the polymerisations (Fig. S3-S11†) were another proof for the high control and reproducibility of the reactions. Neither monomer-consuming side reactions nor the formation of unwanted PEEGE homopolymer in the case of the PBO- $b$-PEEGE syntheses could be observed.

\subsection{Characterisation of atactic and isotactic homopolymers and BCPs}

Structural characterisation. ${ }^{1} \mathrm{H}$ NMR spectra revealed the degrees of polymerisation (DPs), molecular weights and hydrophilic block ratios $f$ (molecular weight of the hydrophilic block divided by the molecular weight of the BCP) of all polymers. An exemplary spectrum of the $(S)$-BCP is presented in Fig. 2; the spectra of all other polymers are shown in Fig. S12-S20.† Three sets of similar PBO- $b$-PG BCPs, PBO and PG homopolymers were obtained, which possessed highly comparable DPs among themselves (Table 1). The DPs of the PBO homopolymers were 27 , of the PG homopolymers $28-30$, and of the BCPs $26-27$ for the PBO blocks and 14 for the PG blocks. The $f$-ratios of the BCPs of $34-35 \%$ were in the range where vesicular structures were reported for atactic PBO- $b$-PGs when subjected to aqueous self-assembly. ${ }^{25}$ Exemplary SEC traces and their dispersities are shown in the inset in Fig. 2 for the $(S)$ PBO homopolymer, $(S)$-PG homopolymer and $(S)$-BCP. The chain extension from the $(S)$-PBO precursor towards the $(S)$ BCP led to a shift to a lower elution volume. The $(S)$-PG homopolymer eluted at even lower volumes, despite having a lower molecular weight than the $(S)$-BCP, which indicates a greater solvodynamic volume of PG compared to PBO. For all polymers, the SEC traces, molecular weights and dispersities are reported in Fig. S21. $\dagger$ Narrow distributions with dispersities between 1.06 and 1.08 were obtained for all nine polymers. Similar solvodynamic volumes between polymers of the same molecular weight but with different configurations suggested that isotactic polymers did not adapt different conformations in DMF solution than atactic polymers. The formation of secondary structures such as helices in organic solvents thus seemed unlikely.

Quantification of meso diads. ${ }^{1} \mathrm{H}$ NMR spectroscopy did not allow for a quantification of the isotactic content of the polymers, as an error-free integration of the respective signals B, C, F and G (Fig. 2) was not possible. Hence, the quantitative determination was done by ${ }^{13} \mathrm{C}$ NMR spectroscopy (Fig. S12$\mathrm{S} 20 \dagger$ ) as commonly done for isotactic polyethers. ${ }^{29-34}$ Integration of the meso $(\mathrm{m})$ and racemic $(r)$ signals of the methylene groups adjacent to the chiral methine carbon led to the relative amount of $m$ conjunctions (Fig. $3 \mathrm{a}, \mathrm{b}$ and S22 $\dagger$ ). ${ }^{35}$ For the polymers synthesised from racemic monomers, $(R / S)$ PBO, $(R / S)$-PG and both blocks of the $(R / S)$-BCP, meso diad contents of $48-55 \% m$ were found (Tables 1 and S1†). Those numbers indicated a statistical distribution of isotactic and syndiotactic conjunctions and confirmed the presence of atactic polymers. For the $(R)$ - and $(S)$-PG homopolymers and the PG blocks of the $(R)$ - and $(S)$-BCPs, $97-99 \% m$ was found, indicating a high excess of isotactic conjunctions. The $(R)$ - and $(S)$-PBO homopolymers and the PBO blocks in the $(R)$ - and $(S)$ - 


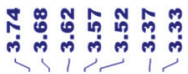

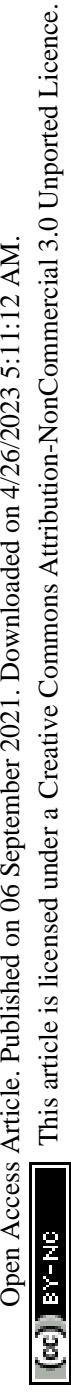

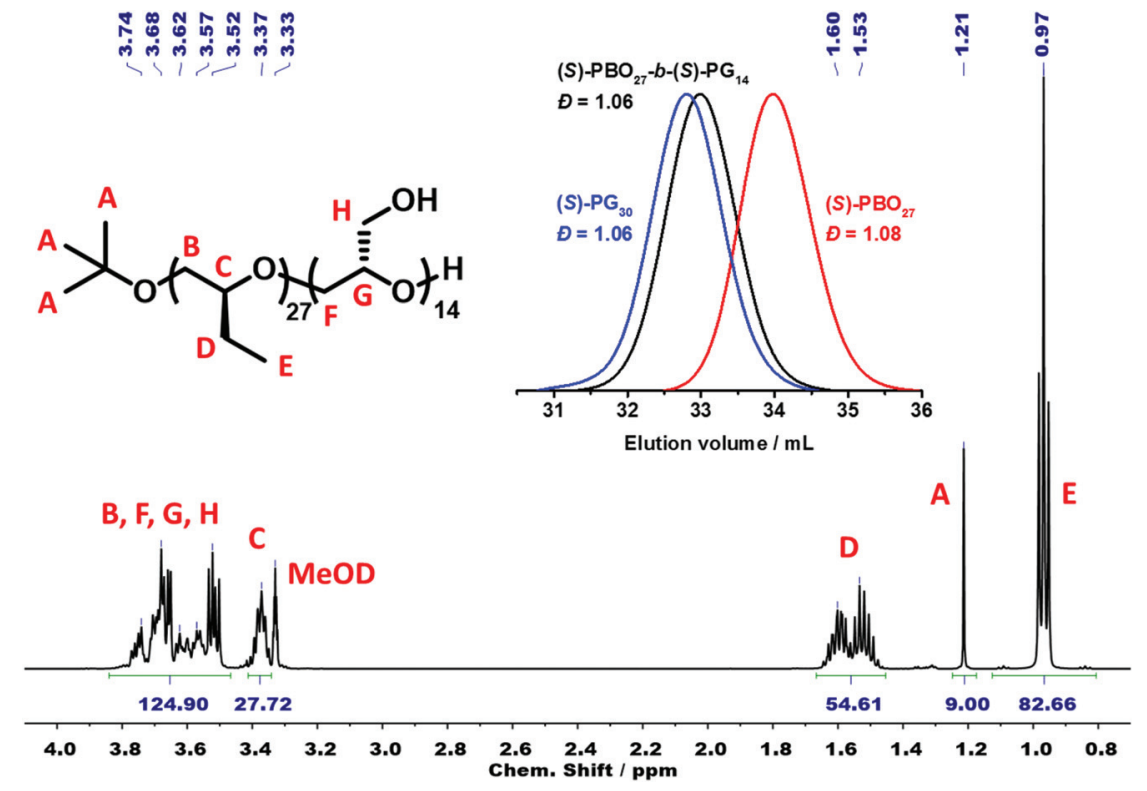

Fig. 2 Structural characterisation of the (S)-BCP (S)-PBO $27-b-(S)-\mathrm{PG}_{14}$ by ${ }^{1} \mathrm{H}$ NMR spectroscopy in methanol- $\mathrm{d}_{4}$. Signal A was used as integration reference and allowed the calculation of the DPs of the PBO block (signal D) and the PG block (signals B, F, G, H). The inset shows the SEC traces, measured in DMF, and the dispersities of the (S)-BCP (black), the (S)-PBO precursor (red) as well as the (S)-PG homopolymer (blue).
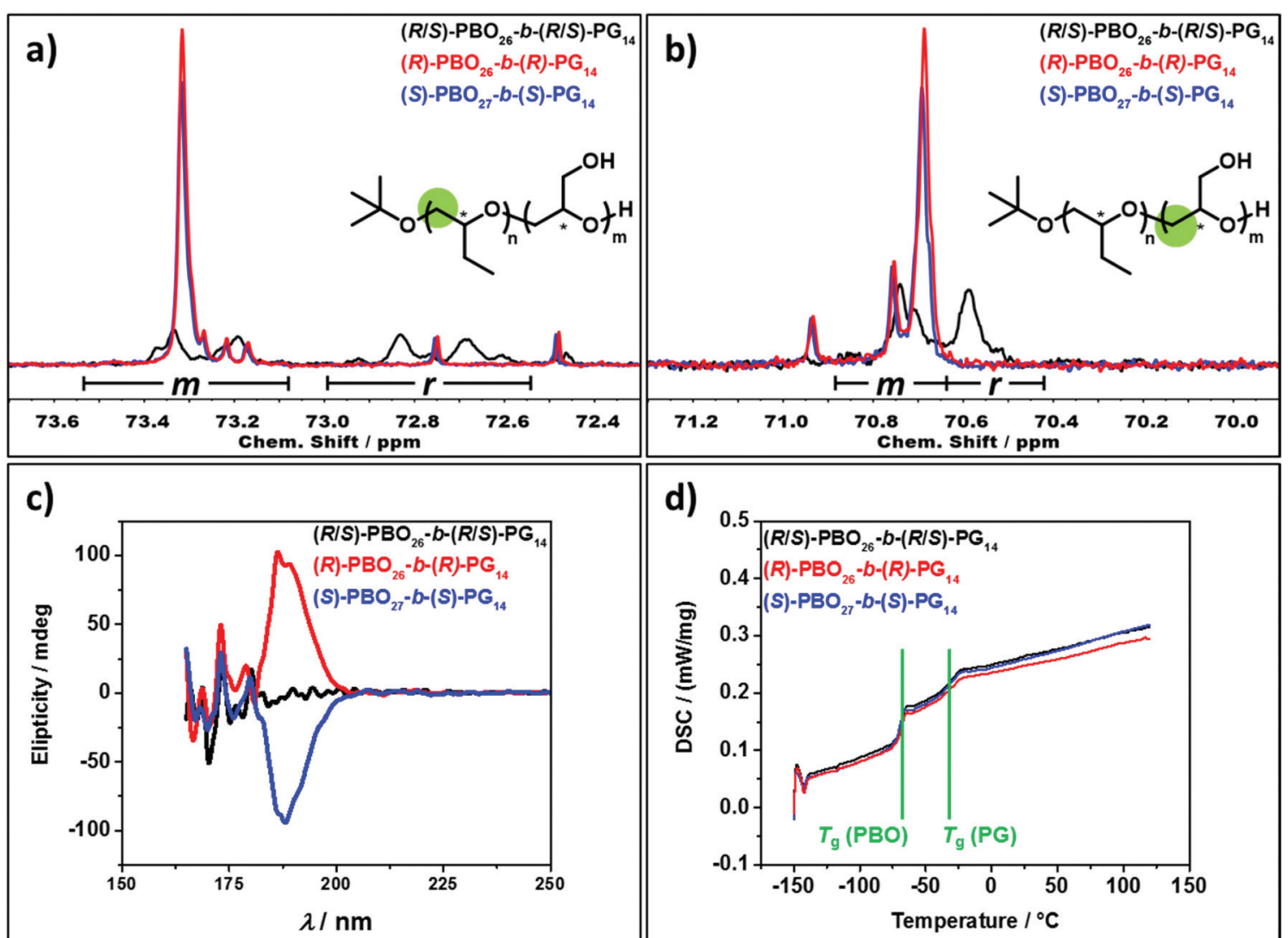

Fig. 3 Chiral characterisation of the atactic and isotactic BCPs. ${ }^{13} \mathrm{C}$ NMR signals of the methylene carbon atoms (highlighted in green) of (a) the PBO block and (b) the PG block. The integrated areas correspond to the racemic $(r)$ and meso $(m)$ diads. (c) CD spectra, measured in $n$-hexane. (d) DSC thermograms with indicated glass transition temperatures $\left(T_{\mathrm{g}}\right)$ of the PBO and PG blocks. 
BCPs showed lower values of $88-91 \% \mathrm{~m}$. Two possible explanations for the lower stereoregularity of the PBO blocks seemed reasonable: a lower $e e$ of the enantiopure BO monomers (Fig. S1 $\dagger$ ) or a more frequent attack on the tertiary epoxide carbon bearing the ethyl side group, as this provides less sterically hindrance compared to the more spacious acetal side group of the EEGE monomers. The latter effect would lead to a loss of the stereoregularity as well as to a loss of the regioregularity, i.e. to an inverted repeating unit (head-head or tail-tail connection). ${ }^{29,32}$ In general, the polymerisations retained the configuration of both enantiopure monomers sufficiently well to refer to the resulting polymers as isotactic (i.e. stereoregular and regioregular). These results are in line with literature values of around $90 \% \mathrm{~m}$ for isotactic poly(propylene oxide) (PPO) synthesised in a similar manner. ${ }^{34}$

Optical characterisation. In order to evaluate the optical properties of all compounds, polarimetry and CD spectroscopy measurements were performed (Tables 1 and S2, Fig. 3c and $\mathrm{S} 23 \dagger)$. Racemic monomers and atactic polymers showed specific rotations close to 0 , in contrast to enantiopure monomers and isotactic polymers. Whilst the magnitude differed between compounds of one configuration, the direction always remained the same: all $(R)$ compounds showed positive, all $(S)$ compounds negative specific rotations. Similar compounds with opposite configuration showed specific rotations in the same order of magnitude (Tables 1 and S2 $\dagger$ ). CD-spectra of all polymers showed absorptions only in the range between 180 and $205 \mathrm{~nm}$. None of the atactic polymers were $\mathrm{CD}$ active (Fig. 3c and $\mathrm{S} 23 \dagger$ ), in contrast to isotactic polymers. Polymers with $(R)$ configuration showed positive signals, whereas polymers with $(S)$ configuration showed negative mirror signals of similar shape and intensity. Structurally induced phenomena such as Cotton effects were not visible, providing further evidence for the absence of any kind of secondary structure of the polymers in solution. ${ }^{36}$ Thus, polarimetry and CD spectroscopy confirmed the presence of optically active isotactic polymers.

Thermal characterisation. To study the thermal properties and obtain further structural insights into the polymers, TGA and DSC measurements were performed. TGA thermograms of the BCPs showed the thermal decomposition at temperatures higher than $250{ }^{\circ} \mathrm{C}$ as a one-step process, independent of their configuration (Fig. S24†). DSC measurements were performed for all BCPs and homopolymers. Glass transitions were measured for all homopolymers, for PBO at about $-75^{\circ} \mathrm{C}$, for PG at about $-30{ }^{\circ} \mathrm{C}$ (Fig. S25 $\dagger$ and Table 1). These numbers were independent of the configuration and in line with literature values for both homopolymers. ${ }^{27,37,38}$ The thermograms of the three BCPs showed two glass transitions at about $-70{ }^{\circ} \mathrm{C}$ and $-30{ }^{\circ} \mathrm{C}$ (Fig. $3 \mathrm{~d}$ and Table 1 ), in line with the ones measured for the respective homopolymers. The slightly lower $T_{\mathrm{g}}$ values of the PBO homopolymers compared to the PBO block in the BCPs could be explained by a less ordered chain packing because of the attached PG block and is literatureknown. ${ }^{25,39}$ Within the measurement accuracy, the $T_{\mathrm{g}}$ values were similar for all configurations. No first order transitions, i.e. melting or crystallisation, could be detected in any traces, confirming the presence of fully amorphous polymers. As for PG this is in contrast to the popular hydrophilic polyether PEG, which is semi-crystalline. ${ }^{40}$ With respect to $\mathrm{PBO}$, the low $T_{\mathrm{g}}$ and the absence of crystallinity should generally allow the insertion of membrane proteins into vesicles containing $\mathrm{PBO}$ as hydrophobic block. With this, the isotactic PBO blocks are in striking contrast to hydrophobic polymers that exhibit glass transition or melting above body temperature or (semi-) crystallinity depending on their tacticity or thermal history, such as PLA, PMMA, PS, PP or PPO. ${ }^{11,40-43}$ Thus, PBO represents the polymer of choice to analyse stereospecific interactions such as membrane protein insertion into chiral, yet fully amorphous self-assembly membranes.

\subsection{Self-assembly into SUVs}

The three BCPs were subjected to the solvent switch protocol established for atactic PBO- $b$-PGs. ${ }^{25}$ After self-assembly in PBS, the structures were characterised using a combination of DLS, SLS, NTA, TEM and Cryo-TEM. Extrusion finally led to more homogenous and narrowly distributed SUV sizes. Extrusion was performed in order to exclude the presence of larger particles above $100 \mathrm{~nm}$ in diameter, as these would hamper a possible cell uptake and future biomedical applications. ${ }^{44}$

Before extrusion, TEM imaging revealed the presence of round shaped self-assemblies for all BCPs (Fig. S26†), indicating vesicular morphologies. Although a high variation in their sizes was observed, homogenous phases with few aggregates and micelles were obtained. DLS and SLS were employed to confirm the presence of SUVs (Table 2). The sizes of all selfassemblies of the three BCPs were in a similar range with hydrodynamic radii $\left(R_{\mathrm{h}}\right)$ between $73 \mathrm{~nm}$ and $98 \mathrm{~nm}$. The $R_{\mathrm{h}}$ values decreased only slightly over the whole angle range, indicating homogenous phases and low polydispersities (Fig. S27$\mathrm{S} 29 \dagger)$. Radii of gyration $\left(R_{\mathrm{g}}\right)$ between $89 \mathrm{~nm}$ and $97 \mathrm{~nm}$ were of a similar magnitude as the $R_{\mathrm{h}}$ values (Table 2), which led to $\rho$ values around 1.0. The shape parameter $\rho=R_{\mathrm{g}} / R_{\mathrm{h}}$ is characteristic for specific morphologies in solution and a value of $\rho=$ 1.0 corresponds to hollow spheres with a thin shell. ${ }^{45,46}$ Values of 0.91 for the $(R / S)$-BCP, 1.29 for the $(R)$-BCP and 1.01 for the $(S)$-BCP confirmed the presence of hollow vesicles. The deviation of the calculated values from 1.0 could be attributed to the lack of extrusion, the polydispersity (PDIs around 0.25 for all BCPs self-assemblies) and that the average size was just inbetween the Guinier and Mie regimes, preventing a more accurate determination of $R_{\mathrm{g}}$. Every BCP was subjected to several repetition runs, where the radii typically varied by $\pm 20 \mathrm{~nm}$, indicating that the SUVs of all three BCPs were formed reproducibly in comparable sizes, independently of their configuration.

After extrusion through a $100 \mathrm{~nm}$ membrane, TEM images indicated the presence of smaller SUVs with diameters between 50 and $200 \mathrm{~nm}$ in homogenous phases (Fig. 4). The size variation of the SUVs had visually decreased compared to the SUVs before extrusion. According to DLS and SLS, the sizes of the SUVs were reduced to radii $\left(R_{\mathrm{h}}\right.$ and $\left.R_{\mathrm{g}}\right)$ between 49 and 
Table 2 Characterisation of the nanoscopic self-assemblies before and after extrusion with a $100 \mathrm{~nm}$ membrane consisting of hydrodynamic radius $\left(R_{\mathrm{h}}\right)$, radius of gyration $\left(R_{\mathrm{g}}\right)$, shape parameter $\rho$ and membrane thickness $l$

\begin{tabular}{|c|c|c|c|c|c|c|c|c|}
\hline Extrusion & Polymer & $R_{\mathrm{h}}{ }^{a} / \mathrm{nm}$ & $\mathrm{PDI}^{a}$ & $R_{\mathrm{g}}{ }^{b} / \mathrm{nm}$ & $\rho=R_{\mathrm{g}}{ }^{b} / R_{\mathrm{h}}{ }^{a}$ & $R_{\mathrm{h}}^{c} / \mathrm{nm}$ & Morphology & $l^{d} / \mathrm{nm}$ \\
\hline- & $(R)-\mathrm{BCP}$ & $73 \pm 8$ & $0.26 \pm 0.03$ & $94 \pm 3$ & $1.29 \pm 0.14$ & - & SUV & - \\
\hline $100 \mathrm{~nm}$ & $(R / S)-\mathrm{BCP}$ & $59 \pm 2$ & $0.26 \pm 0.05$ & $64 \pm 5$ & $1.08 \pm 0.09$ & $56 \pm 11$ & SUV & $11.1 \pm 0.8$ \\
\hline $100 \mathrm{~nm}$ & $(R)-\mathrm{BCP}$ & $49 \pm 3$ & $0.24 \pm 0.04$ & $53 \pm 8$ & $1.08 \pm 0.17$ & $48 \pm 11$ & SUV & $11.6 \pm 0.9$ \\
\hline $100 \mathrm{~nm}$ & $(S)$-ВСР & $64 \pm 2$ & $0.22 \pm 0.04$ & $69 \pm 3$ & $1.08 \pm 0.06$ & $63 \pm 10$ & SUV & $11.0 \pm 1.0$ \\
\hline
\end{tabular}

${ }^{a}$ Values determined by DLS. ${ }^{b}$ Values determined by SLS. ${ }^{c}$ Values determined by NTA. ${ }^{d}$ Values determined by Cryo-TEM.
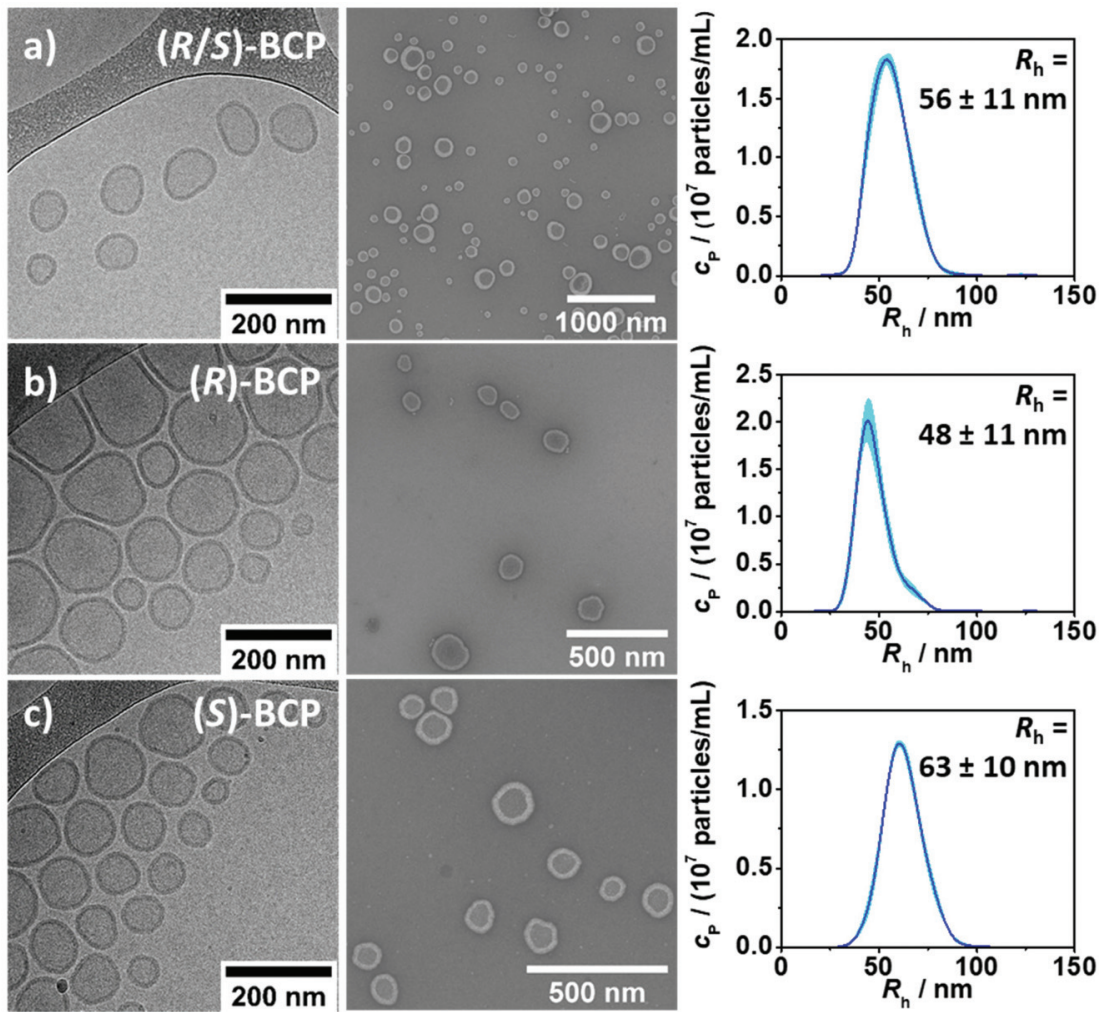

Fig. 4 Characterisation of the SUVs formed by (a) $(R / S)-\mathrm{PBO}_{26}-b-(R / S)-\mathrm{PG}_{14}$, (b) $(R)-\mathrm{PBO}_{26}-b-(R)-\mathrm{PG}_{14}$ and (c) (S)-PBO $27-b-(S)-\mathrm{PG}_{14}$. Left column: Cryo-TEM images; Middle column: TEM images; Right column: Size distributions and average hydrodynamic radii measured by NTA.

$68 \mathrm{~nm}$ (Table 2 and Fig. S30-S32†). The lower measurement errors after extrusion indicated that the homogeneity was increased. The PDIs, however, decreased only negligibly to values between 0.22 and 0.26 (Table 2). The shape parameter remained with $\rho=1.08$ for all three BCPs in a range typical for vesicular structures. In addition, NTA measurements (Fig. 4) were performed to confirm the size distributions. In all cases, monomodal distributions with mean $R_{\mathrm{h}}$ values between 48 and $63 \mathrm{~nm}$ were obtained, being perfectly in line with the DLS results (Table 2). Cryo-TEM imaging (Fig. 4) again confirmed the presence of SUVs and allowed for the determination of the membrane thicknesses $l$. For all BCPs, SUVs with diameters between 45 and $200 \mathrm{~nm}$ were observed. The membrane thicknesses were determined to be $11.1 \pm 0.8 \mathrm{~nm}$ for the $(R / S)$-BCP,
$11.6 \pm 0.9$ for the $(R)$-BCP and $11.0 \pm 1.0$ for the $(S)$-BCP (Table 2). Besides some spherical micelles (Fig. S33†), no other morphologies were visible, highlighting the stability of the vesicular morphology and the flexibility of the membrane. Extrusion allowed for the reproducible formation of highly homogeneous SUVs in terms of size, shape and membrane thickness. Considering the actual membrane thickness and the contour length in a hypothetical stretched conformation as well as the end-to-end distance in an ideal random coil, the degree of the stretching of the PBO block could be calculated (ESI $\dagger$ ). Accordingly, in the $(R / S)$-BCP PBO was $51 \%$ stretched, which is a higher value than observed for more hydrophobic polymers, suggesting less intercoiling within the membrane. ${ }^{47}$ 
SUVs of the $(R / S)$-BCP and $(S)$-BCP remained stable for at least one week at room temperature. In contrast, SUVs composed of the $(R)$-BCP ruptured and formed wormlike aggregates to a small extent (Fig. S34 $\dagger$ ). Due to the structural similarity of the BCPs (Table 1), this instability hinted towards a correlation between the partial loss of long-term stability and the $(R)$ configuration of the BCP. However, it cannot be ascribed to it unequivocally. Hence, it is assumed that SUVs composed of the $(R)$-BCP were not fully at thermodynamic equilibrium. ${ }^{48}$

\subsection{Formation of GUVs by double emulsion microfluidics}

To elucidate the ability of all three BCPs to form microscopic GUVs, a microfluidics-based approach was chosen. Compared to film rehydration, ${ }^{23,49,50}$ GUVs formed by double emulsion microfluidics promise higher reproducibility, size uniformity and encapsulation efficiency. ${ }^{22,51}$ The detailed description of the used microfluidics device, as well as the general concept of this method, can be found elsewhere. ${ }^{22}$ In order to prevent vesicle rupture upon evaporation of the organic solvents, optimising the contents and fluid properties (e.g. viscosity and density) of the inside and outside of GUVs was essential (see Experimental section 2.3). ${ }^{22}$

GUVs were imaged by CLSM and exemplary micrographs of the GUVs composed of all three BCPs are shown in Fig. 5a-c. The GUVs were formed in a high homogeneity with similar sizes: for the $(R / S)$-BCP a GUV diameter of $39.5 \pm 0.5 \mu \mathrm{m}$ was determined, for the $(R)$-BCP $37.3 \pm 1.0 \mu \mathrm{m}$ and for the $(S)$-BCP $35.4 \pm 0.7 \mu \mathrm{m}$. Small deviations were due to necessary slight variations in the optimal flow rate. To improve the visualisa-
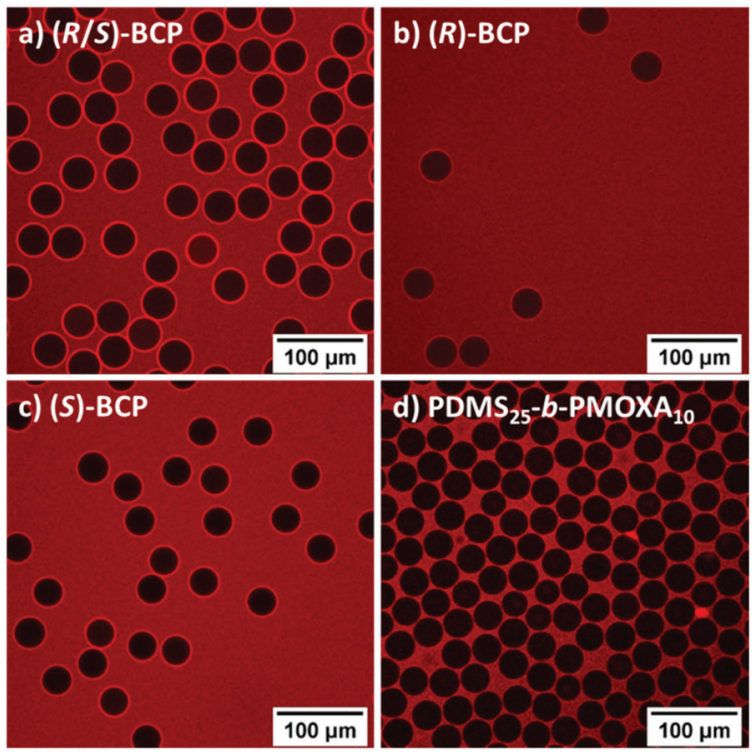

Fig. 5 CLSM images of GUVs composed of (a) $(R / S)-\mathrm{PBO}_{26}-b-(R / S)$ $\mathrm{PG}_{14}$, (b) (R)-PBO $26-b-(R)-\mathrm{PG}_{14}$, (c) (S)-PBO $27-b-(S)-\mathrm{PG}_{14}$ and (d) $\mathrm{PDMS}_{25}-b-\mathrm{PMOXA}_{10}$ formed by double emulsion microfluidics. The red fluorescent dye BODIPY 630/650 was added after GUV formation and stained the membrane and the background, but not the lumen of the GUVs. tion the hydrophobic dye BODIPY 630/650 was added after GUV formation. As the dye was also soluble in PBS, not only the hydrophobic membrane but also the background appeared red (Fig. 5). The lumen of all GUVs remained dark and indicated the successful formation of impermeable GUVs. Differences between the three BCPs were found regarding the time needed for the formation of GUVs from double emulsions and the stability of GUVs. Less than $30 \mathrm{~min}$ were required for the $(R / S)$-BCP and the $(R)$-BCP to form GUVs. In contrast, double emulsions formed by the $(S)$-BCP were present even after three hours. While multiple $(R / S)$-BCP GUVs were stable over several hours, the majority of GUVs composed of the $(R)$ and $(S)$-BCPs disassembled within some minutes upon evaporation of the organic solvents. Consequently, only few GUVs of these BCPs were visible in the CLSM images (Fig. 5b and c).

As a comparison for the GUV stability, GUVs composed of poly(dimethylsiloxane)-block-poly(2-methyl-2-oxazoline)

$\left(\mathrm{PDMS}_{25}-b-\mathrm{PMOXA}_{10}\right)$ of similar molecular weight $(2850 \mathrm{~g}$ $\left.\mathrm{mol}^{-1}, Ð=1.49, f=30 \%\right)^{21}$ were produced. PDMS- $b$-PMOXA based BCPs represent the gold standard for the fabrication of SUVs and GUVs ${ }^{21,49}$ and have recently been shown to form stable and impermeable GUVs by microfluidics. ${ }^{22}$ GUVs composed of $\mathrm{PDMS}_{25}-b$-PMOXA 10 and stained with BODIPY 630/ 650 are shown in Fig. 5d. The diameter was similar to PBO- $b$ PG GUVs and determined to be $34.7 \pm 1.7 \mu \mathrm{m}$. In contrast to the PBO- $b$-PG GUVs only a negligible number of PDMS- $b$ PMOXA GUVs ruptured during formation or within the next days, explaining the high concentration of GUVs in Fig. 5d. The lower stability of PBO- $b$-PG GUVs was in line with the high degree of stretching of $\mathrm{PBO}$ within the membrane, resulting in less intercoiling that could stabilise the membrane. Increasing the membrane thickness could have improved the stability, but would have hindered the desired membrane protein insertion. $^{52}$

\subsection{OmpF insertion and enzyme reaction}

The formation of GUVs set the basis to prove the functional reconstitution of a membrane protein in PBO- $b$-PG membranes. Previously, the microfluidics-based approach allowed to analyse cascade reactions within GUVs equipped with membrane proteins. ${ }^{22}$ Here, an adapted and simplified approach was employed. The outer membrane protein $\mathrm{F}(\mathrm{OmpF})$ from E. coli was chosen as exemplary membrane channel porin since it allows the passage of molecules up to $600 \mathrm{Da}$ (ref. 53) and has been reconstituted in various studies in PDMS- $b$ PMOXA SUV and GUV membranes. ${ }^{21,22}$ As proof of concept, a model enzyme reaction based on the enzyme $\beta$-galactosidase $(\beta$-Gal) was performed (Fig. 6a). $\beta$-Gal cleaves the non-fluorescent substrate resorufin $\beta$-D-galactopyranoside (RGP) into D-galactose and the fluorescent dye resorufin. ${ }^{54} \beta$-Gal was added to the inner aqueous phase (IA) and encapsulated inside the GUVs. For creating the microreactor, OmpF was added to the outer aqueous phase (OA) during double emulsion formation. The negative control was run using similar IA and OA compositions but without OmpF. After GUV formation, proteinase $\mathrm{K}$ was added to deactivate released $\beta$-Gal, originat- 
a)
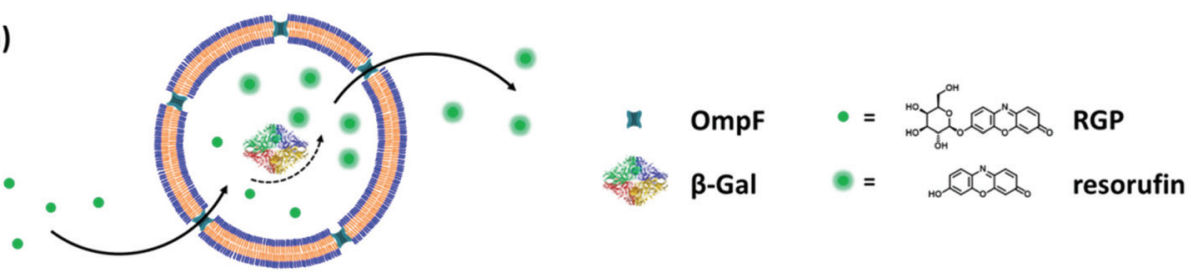

b) $(R / S)-B C P$ with $O \mathrm{mpF}$

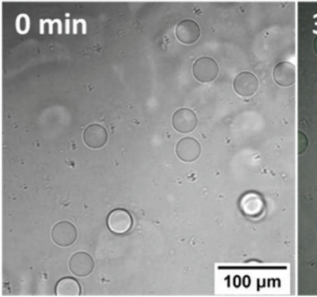

$30 \mathrm{~min}$

c) $(R / S)$-BCP without OmpF

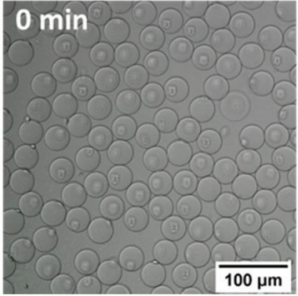

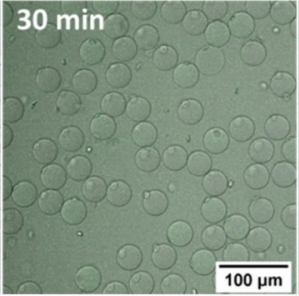
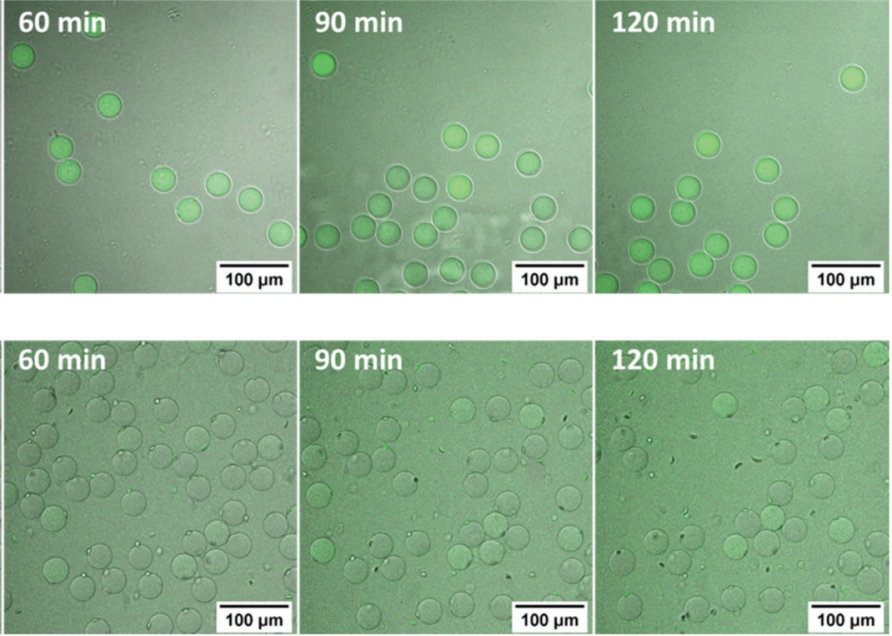

Fig. 6 (a) Schematic representation of the reaction of $\beta$-galactosidase ( $\beta$-Gal) with resorufin $\beta$-D-galactopyranoside (RGP) inside OmpF equipped GUVs (microreactors) to yield the fluorescent dye resorufin. The dye accumulates within the cavity and over time diffuses out of the GUV through the OmpF pores. Snapshots of the enzyme reaction within the cavity of $(R / S)$-BCP GUVs (b) with and (c) without reconstituted membrane protein OmpF, recorded by CLSM at different time points.

ing from a significant amount of GUVs rupturing during formation, before RGP addition or during the two hours of the experiment. Subsequently, RGP was added and the fluorescence development was recorded in a time lapse of two hours by CLSM. Due to the instability of GUVs formed by the isotactic BCP, it was only possible to run this experiment for the atactic $(R / S)$-BCP (videos in ESI $\dagger$ ). Snapshots of the videos after every 30 min are displayed in Fig. $6 \mathrm{~b}$ and c.

In both cases, with and without OmpF, an increased fluorescence over time was visible inside and outside of GUVs. For the microreactor with OmpF (Fig. 6b), the fluorescence inside GUVs increased significantly quicker than the background outside of GUVs, whereas for the negative control (Fig. 6c), no significant difference between inside and outside intensity could be observed. In order to confirm the visual impressions, the mean fluorescence intensities on the inside and the outside of GUVs were measured every 15 min (Fig. 7). The averaged intensities and their standard deviations are displayed in Fig. 7a for the $(R / S)$-BCP microreactor with $\mathrm{OmpF}$ and for the negative control. The intensities of the negative control (inside of GUVs without OmpF, outside of GUVs without OmpF) as well as outside of GUVs with OmpF were similar for all instants of time. Solely the intensity inside GUVs with reconstituted OmpF rose significantly above the background and reached a plateau after $75 \mathrm{~min}$. This behaviour can be interpreted as follows: in case of GUVs with reconstituted OmpF, RGP diffused through the OmpF pores into the GUVs and got cleaved by $\beta$-Gal, forming the fluorescent resorufin, which accumulated inside the cavity and led to a strong increase in fluorescence. Over time, resorufin diffused out of the GUVs through the OmpF pores, leading to an increasing background fluorescence. The development of the plateau after $75 \mathrm{~min}$ indicated a dynamic equilibrium between RGP entering the vesicles and resorufin leaving the inner cavity. When OmpF was missing, the passage of RGP through the membrane was hindered due to the lacking OmpF pores. This behaviour is known in literature for similar microreactors equipped with OmpF. ${ }^{49}$ The background fluorescence of the negative control originated from ruptured GUVs releasing $\beta$-Gal and inducing an enzyme reaction before being cleaved by proteinase $\mathrm{K}$.

In order to confirm the applicability of PBO- $b$-PG microreactors as platform to prove the successful membrane protein insertion, a comparison with the aforementioned $\mathrm{PDMS}_{25}-b$ PMOXA $_{10}$ GUVs was drawn. This polymer was already employed to reconstitute OmpF in its membrane ${ }^{21}$ and has a comparable membrane thickness $(12.0 \pm 0.8 \mathrm{~nm}$, Fig. S35†) to the one of the $(R / S)$-BCP. The videos and snapshots of the enzyme reaction can be found in the ESI (Fig. S36†). The PDMS- $b$-PMOXA based microreactors exhibited a similar behaviour as the $(R / S)$-BCP GUVs regarding the intensity development inside and outside of GUVs with and without OmpF (Fig. 7b). In contrast to $(R / S)$-BCP GUVs, no plateau but rather a constant increase in the fluorescence intensity was observed for $\mathrm{PDMS}_{25}-b-\mathrm{PMOXA}_{10}$ GUVs. As the intensity plateau was 
a) $(R / S)-\mathrm{BCP}$
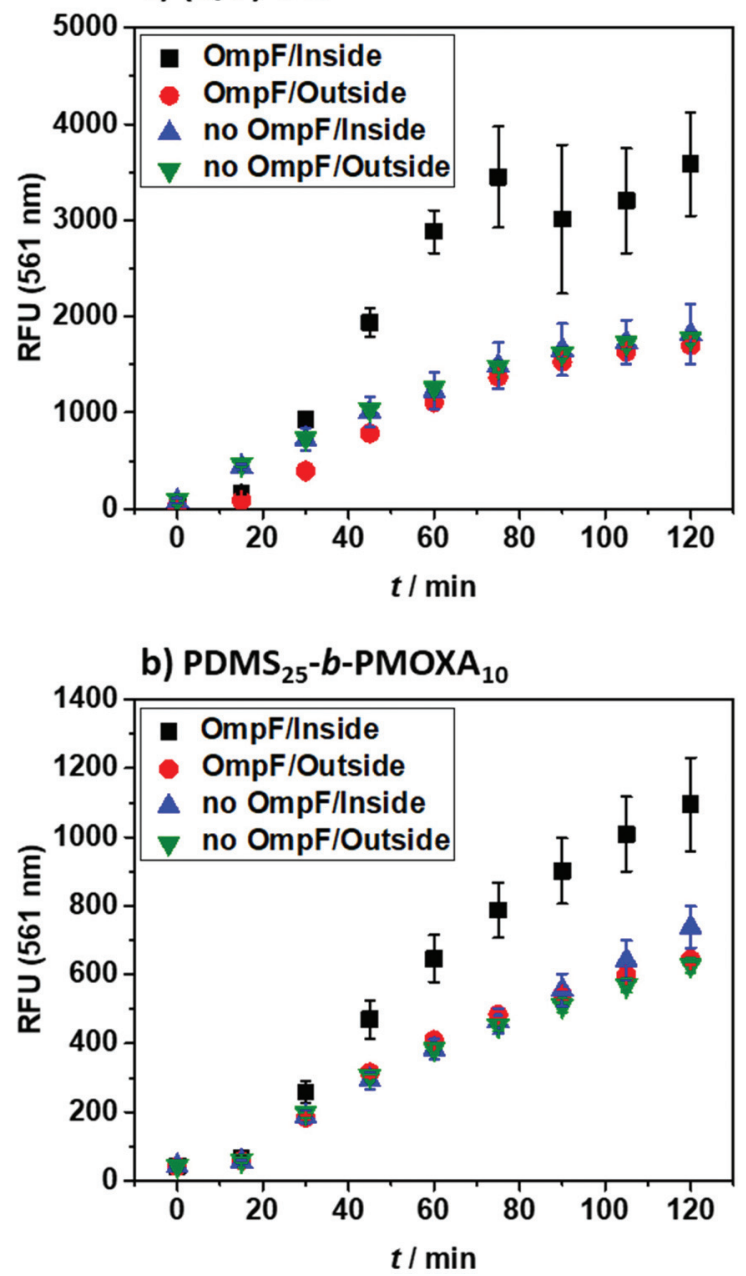

Fig. 7 Fluorescence intensity development of (a) $(R / S)-B C P$ and (b) $\mathrm{PDMS}_{25}-b-\mathrm{PMOXA}_{10}$ GUVs resulting from the reaction of resorufin $\beta$-Dgalactopyranoside (RGP) with $\beta$-galactosidase ( $\beta$-Gal) to yield fluorescent resorufin. The intensities measured inside GUVs containing OmpF (black squares), outside of GUVs containing OmpF (red circles), inside of GUVs without OmpF (blue triangles) and outside of GUVs without OmpF (green triangles) are displayed. Up to 15 GUVs per image were measured and the mean intensities were calculated using their standard deviations as errors.

reached earlier for $(R / S)$-BCP microreactors, a higher permeability was hinted for PBO- $b$-PG membranes than for PDMS$b$-PMOXA membranes. Similarly, the negative control of $(R / S)$ BCP-based GUVs led to a higher background fluorescence than in case of PDMS- $b$-PMOXA GUVs, which also indicated a higher permeability of PBO- $b$-PG membranes. This was coherent with literature as the latter are known to form highly impermeable membranes due to their high hydrophobicity. ${ }^{18,20,55}$ To conclude, both BCPs were equally able to reconstitute OmpF. Thus, the $\mathrm{PBO}-b$-PG system sets the first basis to analyse the insertion of membrane proteins into chiral membranes composed of isotactic PBO- $b$-PG. In turn, this could allow for significantly faster reaction rates than with membranes from PDMS- $b$-PMOXA, once the GUVs are sufficiently stable.

\section{Conclusion}

This work represents the first study discriminating the effect of tacticity from crystallinity in aqueous self-assemblies of amphiphilic BCPs. Three PBO- $b$-PG BCPs were synthesised, differing solely in their configuration: one atactic $(R / S)$-BCP, one isotactic $(R)$-BCP and one isotactic $(S)$-BCP. All polymerisations were highly controlled and showed perfect first-order kinetics. Tacticity and optical activity were proven by polarimetry, CD spectroscopy and ${ }^{13}$ C-NMR spectroscopy. Despite the differences in their respective stereochemistry, all polymers exhibited similar thermal and structural behaviour, proving that the stereoregularity did not induce crystallinity or the formation of secondary structures in bulk or in solution. Subsequently, all BCPs were self-assembled in aqueous medium into homogenous phases of SUVs and GUVs. The potential of fully amorphous PBO- $b$-PG membranes for complex applications involving membrane proteins was highlighted by a successful and functional reconstitution of the membrane protein OmpF into $(R / S)$-BCP GUVs, allowing for the creation of a microreactor to perform enzyme reactions. Stability differences between $(R / S)$-BCP, $(R)$-BCP and $(S)$-BCP were found for SUVs and GUVs, suggesting an influence of the tacticity of the respective BCPs. Expending the BCP library to include blocks with altering configuration (e.g. $(R)$ PBO- $b-(S)-\mathrm{PG}, \quad(S)-\mathrm{PBO}-b-(R)-\mathrm{PG})$ could be interesting to analyse the effects of the configuration of every block solely. However, following the rather small differences between $(R)$ and $(S)$-BCPs discovered in this work, it is unlikely that a combination of different configuration has a measureable effect. In general, SUVs composed of atactic and isotactic BCPs were stable at least over one week. However, the BCP composition needs to be further optimised to access more stable GUVs. The ability to reconstitute membrane proteins in fully amorphous isotactic PBO- $b$-PG membranes provides a basis to analyse the interplay of the membrane chirality with specific pores or encapsulated drugs. Such chiral recognition could be beneficial for the directional insertion of transmembrane proteins. This would allow for a unidirectional passage of substrates, which has so far been achieved only by employing asymmetric lipid membranes or ABC triblock copolymer membranes.

\section{Author contributions}

R. W.: Conceptualisation, investigation, project administration, visualisation, writing - original draft. E. C. d. S: Investigation, resources. M. M.: Resources. V. C.: Investigation. J. G.: Conceptualisation, writing - review \& editing. W. M.: Funding acquisition, supervision, writing - review \& editing.

\section{Conflicts of interest}

There are no conflicts to declare. 


\section{Acknowledgements}

The authors thank the Swiss National Science Foundation (SNSF), the National Centre for Competence in ResearchMolecular Systems Engineering (NCCR-MSE) and the University of Basel for financial support. We thank Carola Alampi from BioEM lab (University of Basel) for Cryo-TEM measurements and the Nano Imaging Lab (University of Basel) under Dr Markus Dürrenberger for providing the TEM. Dr Adrian Dinu and Prof. Cornelia Palivan are acknowledged for fruitful discussions and advice. Alain Jeanrenaud is thanked for support with the PG homopolymer synthesis, Sven Freimann for the TGA measurements and Dr Daniel Messmer for proofreading the manuscript.

\section{References}

1 E. Rideau, R. Dimova, P. Schwille, F. R. Wurm and K. Landfester, Chem. Soc. Rev., 2018, 47, 8572-8610.

2 M. Garni, R. Wehr, S. Y. Avsar, C. John, C. Palivan and W. Meier, Eur. Polym. J., 2019, 112, 346-364.

3 X. Zhang, Q. Huang, F. Wang, H. Sun, J. Xiao, E. J. Cornel, Y. Zhu and J. Du, ACS Macro Lett., 2021, 1015-1022.

4 P. Wei, E. J. Cornel and J. Du, Macromolecules, 2021, 54(16), 7603-7611.

5 T. Wang, Y. Li, E. J. Cornel, C. Li and J. Du, ACS Nano, 2021, 15, 9027-9038.

6 Z. Li, R. Liu, B. Mai, S. Feng, Q. Wu, G. Liang, H. Gao and F. Zhu, Polym. Chem., 2013, 4, 954-960.

7 N. Petzetakis, A. P. Dove and R. K. O'Reilly, Chem. Sci., 2011, 2, 955-960.

8 N. Petzetakis, D. Walker, A. P. Dove and R. K. O'Reilly, Soft Matter, 2012, 8, 7408-7414.

9 L. Sun, N. Petzetakis, A. Pitto-Barry, T. L. Schiller, N. Kirby, D. J. Keddie, B. J. Boyd, R. K. O'Reilly and A. P. Dove, Macromolecules, 2013, 46, 9074-9082.

10 A. Das, K. Petkau-Milroy, G. Klerks, B. Van Genabeek, R. P. M. Lafleur, A. R. A. Palmans and E. W. Meijer, ACS Macro Lett., 2018, 7, 546-550.

11 K. Petkau-Milroy, A. Ianiro, M. M. L. Ahn, J. R. Magana, M. E. J. Vleugels, B. A. G. Lamers, R. Tuinier, I. K. Voets, A. R. A. Palmans and E. W. Meijer, ACS Macro Lett., 2020, 9, 38-42.

12 M. E. J. Vleugels, M. E. De Zwart, J. R. Magana, B. A. G. Lamers, I. K. Voets, E. W. Meijer, K. Petkau-Milroy and A. R. A. Palmans, Polym. Chem., 2020, 11, 71707177.

13 F. Itel, M. Chami, A. Najer, S. Lörcher, D. Wu, I. A. Dinu and W. Meier, Macromolecules, 2014, 47, 7588-7596.

14 F. Itel, A. Najer, C. G. Palivan and W. Meier, Nano Lett., 2015, 15, 3871-3878.

15 P. Dimitrov, A. Porjazoska, C. P. Novakov, M. Cvetkovska and C. B. Tsvetanov, Polymer, 2005, 46, 6820-6828.

16 E. V. Konishcheva, U. E. Zhumaev and W. P. Meier, Macromolecules, 2017, 50, 1512-1520.
17 M. Garni, S. Thamboo, C. A. Schoenenberger and C. G. Palivan, Biochim. Biophys. Acta, Biomembr., 2017, 1859, 619-638.

18 A. Belluati, I. Craciun, J. Liu and C. G. Palivan, Biomacromolecules, 2018, 19, 4023-4033.

19 A. Belluati, I. Craciun, C. E. Meyer, S. Rigo and C. G. Palivan, Curr. Opin. Biotechnol., 2019, 60, 53-62.

20 M. Garni, T. Einfalt, R. Goers, C. G. Palivan and W. Meier, ACS Synth. Biol., 2018, 7, 2116-2125.

21 C. E. Meyer, I. Craciun, C. A. Schoenenberger, R. Wehr and C. G. Palivan, Nanoscale, 2021, 13, 66-70.

22 E. C. dos Santos, A. Belluati, D. Necula, D. Scherrer, C. E. Meyer, R. P. Wehr, E. Lörtscher, C. G. Palivan and W. Meier, Adv. Mater., 2020, 32, 1-13.

23 A. Belluati, S. Thamboo, A. Najer, V. Maffeis, C. von Planta, I. Craciun, C. G. Palivan and W. Meier, Adv. Funct. Mater., 2020, 30(32), 2002949-2002959.

24 H. Du, F. A. De Oliveira, L. J. C. Albuquerque, G. Tresset, E. Pavlova, C. Huin, P. Guégan and F. C. Giacomelli, Langmuir, 2020, 36, 1266-1278.

25 R. Wehr, J. Gaitzsch, D. Daubian, C. Fodor and W. Meier, RSC Adv., 2020, 10, 22701-22711.

26 M. Imran ul-haq, B. F. L. Lai, R. Chapanian and J. N. Kizhakkedathu, Biomaterials, 2012, 33, 9135-9147.

27 C. Gerstl, G. J. Schneider, W. Pyckhout-Hintzen, J. Allgaier, D. Richter, A. Alegría and J. Colmenero, Macromolecules, 2010, 43, 4968-4977.

28 A. Thomas, S. S. Müller and H. Frey, Biomacromolecules, 2014, 15, 1935-1954.

29 F. C. Schilling and A. E. Tonelli, Macromolecules, 1986, 19, 1337-1343.

30 B. Wu, C. J. Harlan, R. W. Lenz and A. R. Barron, Macromolecules, 1997, 30, 316-318.

31 B. Antelmann, M. H. Chisholm, S. S. Iyer, J. C. Huffman, D. Navarro-Llobet, M. Pagel, W. J. Simonsick and W. Zhong, Macromolecules, 2001, 34, 3159-3175.

32 M. H. Chisholm and D. Navarro-Llobet, Macromolecules, 2002, 35, 2389-2392.

33 M. A. B. Block and S. Hecht, Macromolecules, 2008, 41, 3219-3227.

34 A. J. McGrath, W. Shi, C. G. Rodriguez, E. J. Kramer, C. J. Hawker and N. A. Lynd, Polym. Chem., 2015, 6, 1465-1473.

35 G. Odian, Principles of Polymerization, John Wiley \& Sons, Inc., Hoboken, NJ, USA, 2004.

36 G. Liu, X. Li, J. Sheng, P.-Z. Li, W. K. Ong, S. Z. F. Phua, H. Ågren, L. Zhu and Y. Zhao, ACS Nano, 2017, 11, 1188011889.

37 F. Wurm, U. Kemmer-Jonas and H. Frey, Polym. Int., 2009, 58, 989-995.

38 F. Wurm, J. Nieberle and H. Frey, Macromolecules, 2008, 41, 1184-1188.

39 C. Lebleu, L. Rodrigues, J. M. Guigner, A. Brûlet, E. Garanger and S. Lecommandoux, Langmuir, 2019, 35, 13364-13374.

40 W. Shi, A. J. McGrath, Y. Li, N. A. Lynd, C. J. Hawker, G. H. Fredrickson and E. J. Kramer, Macromolecules, 2015, 48, 3069-3079. 
41 D. W. van Krevelen and K. te Nijenhuis, Properties of Polymers, Elsevier, 4th edn, 2009.

42 Y. Wang, S. S. Funari and J. F. Mano, Macromol. Chem. Phys., 2006, 207, 1262-1271.

43 J. A. Faucher, J. Polym. Sci., Part B: Polym. Lett., 1965, 3, 143-145.

44 N. Chuard, G. Gasparini, D. Moreau, S. Lörcher, C. Palivan, W. Meier, N. Sakai and S. Matile, Angew. Chem., Int. Ed., 2017, 56, 2947-2950.

45 W. Burchard, Static and dynamic light scattering from branched polymers and biopolymers, in Light Scattering from Polymers. Advances in Polymer Science, Springer, Berlin, Heidelberg, 48th edn, 1983.

46 S. U. Egelhaaf and P. Schurtenberger, J. Phys. Chem., 1994, 98, 8560-8573.

47 D. Daubian, A. Fillion, J. Gaitzsch and W. Meier, Macromolecules, 2020, 53, 11040-11050.

48 M. Dionzou, A. Morère, C. Roux, B. Lonetti, J. D. Marty, C. Mingotaud, P. Joseph, D. Goudounèche, B. Payré,
M. Léonetti and A. F. Mingotaud, Soft Matter, 2016, 12, 2166-2176.

49 M. Garni, T. Einfalt, R. Goers, C. G. Palivan and W. Meier, ACS Synth. Biol., 2018, 7, 2116-2125.

50 S. Thamboo, A. Najer, A. Belluati, C. von Planta, D. Wu, I. Craciun, W. Meier and C. G. Palivan, Adv. Funct. Mater., 2019, 29, 1-12.

51 E. Amstad, S. H. Kim and D. A. Weitz, Angew. Chem., Int. Ed., 2012, 51, 12499-12503.

52 A. Belluati, V. Mikhalevich, S. Yorulmaz Avsar, D. Daubian, I. Craciun, M. Chami, W. P. Meier and C. G. Palivan, Biomacromolecules, 2020, 21, 701-715.

53 G. Kefala, C. Ahn, M. Krupa, L. Esquivies, I. Maslennikov, W. Kwiatkowski and S. Choe, Protein Sci., 2010, 19, 1117-1125.

54 R. B. Liebherr, A. Hutterer, M. J. Mickert, F. C. Vogl, A. Beutner, A. Lechner, H. Hummel and H. H. Gorris, Anal. Bioanal. Chem., 2015, 407, 7443-7452.

55 M. P. Wolf, G. B. Salieb-Beugelaar and P. Hunziker, Prog. Polym. Sci., 2018, 83, 97-134. 